\title{
Competitividade Relativa entre Cultivares de ARROZ IRrigado e Biótipo DE ARROZ-Vermelho ${ }^{1}$
}

\author{
Relative Competitivity among Flooded Rice Cultivars and a Red Rice Biotype
}

FLECK, N.G. ${ }^{2}$, AGOSTINETTO, D. ${ }^{3}$, GALON, L. ${ }^{4}$ e SCHAEDLER, C.E. ${ }^{4}$

\begin{abstract}
RESUMO - O objetivo deste trabalho foi comparar as habilidades competitivas relativas entre dois cultivares de arroz e um biótipo de arroz-vermelho. Para isso, foram realizados experimentos em casa de vegetação na Universidade Federal do Rio Grande do Sul, na estação de crescimento 2001/02. O delineamento experimental utilizado foi o completamente casualizado, com quatro repetições. Os tratamentos foram arranjados em série de substituição e constituíram-se de cinco proporções de plantas de arroz e do biótipo competidor: 100:0, 75:25, 50:50, 25:75 e 0:100. O arroz foi representado pelos cultivares IRGA 417 e EEA 406, e os competidores, pelo arroz-vermelho e pelo cultivar EEA 406, usado como simulador daquele. A análise da competitividade foi efetuada por meio de diagramas aplicados a experimentos substitutivos e uso de indices de competitividade relativa. As variáveis estudadas foram afilhamento, estatura, área foliar e massa seca da parte aérea das plantas. O arroz-vermelho modificou negativamente o número de afilhos, a estatura e a massa seca da parte aérea das plantas dos cultivares IRGA 417 e EEA 406, demonstrando habilidade competitiva superior. Os cultivares de arroz IRGA 417 e EEA 406 não modificaram suas características morfofisiológicas quando em competição, independentemente da proporção de plantas entre ambos, demonstrando habilidades competitivas equivalentes.
\end{abstract}

Palavras-chave: características morfofisiológicas, competição, interferência, plantas daninhas.

\begin{abstract}
The objective of this study was to compare the relative competitive abilities between two rice cultivars and a red rice biotype. Greenhouse experiments were carried out at the Universidade Federal do Rio Grande do Sul, in the 2001/2002 growing season, in a completely randomized design, with four replications. The treatments were arranged in a replacement series assay, with five plant proportions of rice cultivars and competitor biotype being established: 100:0; 75:25; 50:50;25:75 and 0:100, respectively. Rice was represented by the cultivars IRGA 417 and EEA 406, and the competitors by a red rice genotype as well as by EEA 406 cultivar, mimicking red rice. Competitive analysis was accomplished through diagrams usually applied to replacement series studies, plus use of relative competitive indices. The plant variables evaluated were: tillering, plant height, leaf area, and shoot dry mass. Red rice negatively modified plant tillering, plant height, leaf area, and shoot dry mass of both rice cultivars, proving superior competitive ability. The rice cultivars IRGA 417 and EEA 406 did not change their morpho-physiological plant characteristics when in association with each other, regardless of plant proportions among them, presenting equivalent competitive abilities.
\end{abstract}

Keywords: morpho-physiological characteristics, competition, interference, weeds

Recebido para a publicação em 5.6.2007 e na forma revisada em 4.1.2008.

2 Engo-Agr ${ }^{\circ}$., Ph.D. Prof. da Faculdade de Agronomia da Universidade Federal do Rio Grande do Sul - UFRGS, Bolsista em Produtividade de Pesquisa do CNPq; ${ }^{3}$ Engo-Agr ${ }^{\circ}$., Dr., Prof. da Faculdade de Agronomia Eliseu Maciel da Universidade Federal de Pelotas - UFPel, Bolsista em Produtividade de Pesquisa do CNPq; ${ }^{4}$ Engo-Agro ${ }^{\text {., }}$ aluno do Programa de Pós-Graduação em Fitossanidade da UFPel, Bolsista da CAPES. 


\section{INTRODUÇÃO}

A cultura do arroz irrigado assume grande importância no Rio Grande do Sul (RS), pois são cultivados aproximadamente 1,1 milhão de hectares, sendo o Estado responsável por $58,4 \%$ da produção nacional (CONAB, 2006). A produtividade média de grãos obtida na última década no RS foi de $5,5 \mathrm{t} \mathrm{ha}^{-1}$, quase o dobro da média nacional (CONAB, 2006). No entanto, essa produtividade está aquém das obtidas em campos experimentais. Dentre as prováveis causas dessa situação destacam-se: uso de cultivares inadequados e de sementes de baixa qualidade, semeadura fora da época recomendada, manejo inadequado do solo e da água, adubação insuficiente e em época incorreta e, principalmente, controle deficiente de plantas daninhas.

$\mathrm{O}$ arroz-vermelho destaca-se entre as principais espécies daninhas que infestam as lavouras do Estado, por ser a que mais limita o potencial de produtividade do arroz (Souza \& Fischer, 1986). As perdas diretas decorrentes da competição exercida pelo arroz-vermelho são estimadas em cerca de 20\% (Souza \& Fischer, 1986). Já as perdas indiretas, como elevação do custo de produção, depreciação do valor comercial das áreas de cultivo e depreciação comercial do produto colhido, reduzem ainda mais a rentabilidade da lavoura (Menezes \& Silva, 1998). O grau de interferência exercido pelo arroz-vermelho varia com o nível de infestação, as condições edafoclimáticas, as características do cultivar, o período de convivência com a cultura e o biótipo encontrado na área (Agostinetto et al., 2001; Sanchez-Olguín et al., 2007).

As perdas de produtividade das culturas, em decorrência da competição de plantas daninhas, geralmente aumentam quanto mais semelhantes forem suas características morfofisiológicas (Lamego et al., 2004). Para que ocorra competição, há necessidade de sobreposição suficiente dos nichos dos indivíduos que competem, de modo que eles passem a utilizar os mesmos recursos (Maluf, 1999).

As plantas podem competir pelos recursos luz, água, nutrientes e, em algumas situações, também por $\mathrm{CO}_{2}$. A duração da competição determina prejuízos variáveis no crescimento e no desenvolvimento e, conseqüente- mente, na produção das culturas (Christoffoleti \& Victoria Filho, 1996; Lamego et al., 2004; Sanchez-Olguín et al., 2007). O grau de competição depende de fatores relacionados à comunidade infestante (composição, população, distribuição e época de emergência) e à própria cultura (espécie ou cultivar, espaçamento entre linhas e densidade de semeadura).

Dentre os fatores ligados à comunidade infestante, a população de plantas pode ser considerada um dos mais importantes, de forma que, quanto maior a população, maior será a quantidade de indivíduos que disputam os mesmos recursos do meio e mais intensa será a competição sofrida pela cultura (Christoffoleti \& Victória Filho, 1996).

Em áreas agrícolas, a população das plantas cultivadas geralmente é constante, ao passo que a população das espécies daninhas varia em função da quantidade de sementes depositadas no banco do solo, com o nível de infestação e com as condições encontradas no local, em variação na proporção entre as espécies daninhas e a cultura. Desse modo, não basta avaliar a população de plantas no processo competitivo, pois é importante verificar a influência da variação na proporção entre espécies (Christoffoleti \& Victoria Filho, 1996).

A determinação das interações competitivas entre genótipos e espécies de plantas requer delineamentos experimentais e métodos de análise apropriados, sendo os experimentos substitutivos convencionais os mais usados para esclarecer tais relações (Roush et al., 1989).

Em experimentos em séries substitutivas, geralmente as culturas demonstram maior habilidade competitiva do que as espécies daninhas. Isso ocorre porque, em campo, o efeito da planta daninha sobre a cultura se deve, principalmente, à maior população e não à sua habilidade competitiva individual (Vilá et al., 2004). Contudo, quando há competição entre indivíduos do mesmo gênero e/ou espécie, a vantagem da cultura poderá ser alterada, uma vez que ambas exploram o mesmo nicho ecológico. Por pertencerem ao mesmo gênero (Vaughan et al., 2001) e, para a maioria dos autores, à mesma espécie (Pantone \& Baker, 1991; Ogawa, 1992; Fischer \& Ramirez, 1993), o arroz-vermelho e o arroz cultivado provavelmente competem pelo mesmo nicho, o que 
poderá resultar em vantagem competitiva para a planta daninha.

O objetivo deste trabalho foi comparar as habilidades competitivas relativas dos cultivares de arroz IRGA 417 e EEA 406 entre si e em relação ao arroz-vermelho.

\section{MATERIAL E MÉTODOS}

Experimentos foram conduzidos em casa de vegetação na Faculdade de Agronomia da Universidade Federal do Rio Grande do Sul (UFRGS), em Porto Alegre-RS, na estação de cultivo 2001/02. Os ensaios foram alocados em vasos plásticos com capacidade volumétrica de $4 \mathrm{~L}$, preenchidos com solo oriundo de lavoura orizícola, classificado como Planossolo Hidromórfico Eutrófico arênico, pertencente à unidade de mapeamento Vacacaí (EMBRAPA, 1999), e a adubação foi realizada conforme as recomendações para a cultura do arroz irrigado (SOSBAI, 2001). O delineamento experimental foi o completamente casualizado, com quatro repetições. Os competidores incluíram os cultivares de arroz IRGA 417 e EEA 406 e um biótipo de arroz-vermelho.

Primeiramente, realizou-se experimento preliminar com o cultivar de arroz IRGA $417 \mathrm{em}$ monocultivo, visando determinar a população de plantas em que a produção final de massa seca se torna constante. Neste, foram utilizadas populações de 4, 8, 12, 16, 20, 24, 28 e 32 plantas por vaso (equivalentes a 157, 314, 471, $628,785,942,1.099$ e 1.256 plantas $\mathrm{m}^{-2}$ ). A produção final constante da massa seca foi obtida com população de 24 plantas por vaso, equivalente a 942 plantas $\mathrm{m}^{-2}$.

Os experimentos em série de substituição incluíram diferentes combinações dos cultivares de arroz IRGA 417 e EEA 406 e do biótipo de arroz-vermelho, variando-se as proporções relativas de plantas por vaso de 100:0, 75:25, 50:50, 25:75 e 0:100 (0:24, 6:18, 12:12, 18:6 e 24:0), com manutenção da população total de plantas. Para estabelecer as populações desejadas em cada tratamento e obter uniformidade das plântulas, as sementes foram previamente semeadas em bandejas, com posterior transplante para os vasos.

Na primeira combinação de competidores, foram testados os cultivares de arroz IRGA 417 com EEA 406 (este, por apresentar algumas características morfológicas de planta semelhantes às do arroz-vermelho, exerceu a função de simulador). Nas demais combinações, avaliou-se a convivência dos cultivares de arroz IRGA 417 ou EEA 406 com o arroz-vermelho.

Aos 32 dias após a emergência (DAE), avaliaram-se as variáveis: afilhamento (Afi), estatura (Est), área foliar (AF) e massa seca da parte aérea (MS) das plantas. O Afi foi obtido pela contagem do número de afilhos. A Est foi obtida pela mensuração da distância da base até a extremidade da última folha. A quantificação da AF foi realizada com auxílio de integrador eletrônico, em dez plantas coletadas aleatoriamente em cada vaso, ou em todas as plantas nas populações menores. Para se obter a MS, as plantas foram seccionadas ao nível do solo, e a secagem do material foi efetuada em estufa de circulação forçada de ar a $60{ }^{\circ} \mathrm{C}$, até massa constante.

O método da análise gráfica da variação ou produtividade relativa (Radosevich, 1987; Roush et al., 1989; Cousens, 1991) foi utilizado na análise dos dados. O referido procedimento consiste na construção de um diagrama, tendo por base a produtividade relativa (PR) e a produtividade relativa total (PRT).

Quando o resultado da PR tende a uma linha reta, significa que as habilidades das espécies são equivalentes. Caso a PR resulte em linha côncava, indica que existe prejuízo no crescimento de uma ou de ambas as espécies. Ao contrário, se a PR mostrar linha convexa, há benefício no crescimento de uma ou de ambas as espécies. Quando a PRT for igual à unidade (1) (linha reta), ocorre competição pelos mesmos recursos; se ela for superior a 1 (linha convexa), a competição é evitada. Caso a PRT seja menor que 1 (linha côncava), ocorre prejuízo mútuo ao crescimento (Cousens, 1991).

Calcularam-se ainda os indices de competitividade relativa $(\mathrm{CR})$, coeficiente de agrupamento relativo $(\mathrm{K})$ e agressividade (A). A CR representa o crescimento comparativo do genótipo $\mathrm{X}$ em relação ao $\mathrm{Y}$; $\mathrm{K}$ indica a dominância relativa de um genótipo sobre o outro; e A aponta qual dos genótipos é mais agressivo. A interpretação conjunta desses índices indica com 
maior segurança qual genótipo se manifesta mais competitivo (Cousens, 1991). O genótipo $\mathrm{X}$ é mais competitivo que $\mathrm{Y}$ quando $\mathrm{CR}>1, \mathrm{~K}_{\mathrm{x}}$ $>\mathrm{K}_{\mathrm{y}}$ e A > 0; por outro lado, o genótipo $\mathrm{Y}$ é mais competitivo que $\mathrm{X}$ quando $\mathrm{CR}<1, \mathrm{~K}_{\mathrm{x}}<\mathrm{K}_{\mathrm{y}}$ e $\mathrm{A}<$ 0 (Hoffman \& Buhler, 2002). Para calcular esses indices foram usadas as proporções 50:50 das espécies, utilizando-se as seguintes equações, conforme sugerido por Cousens \& O'Neill (1993): $C R=P R_{x} / P R_{y} ; K_{x}=P R_{x} /\left(1-P R_{x}\right) ; K_{y}=P_{y} /$ $\left(1-P R_{y}\right) ; A=P R_{x}-P R_{y}$.

A análise estatística da produtividade ou variação relativa incluiu o cálculo das diferenças para os valores de PR (DPR) obtidos nas proporções de 25, 50 e $75 \%$, em relação aos valores pertencentes à reta hipotética nas respectivas proporções: $0,25,0,50$ e 0,75 para $P R$ (Passini, 2001). Utilizou-se o teste " $t$ " para testar as diferenças relativas aos indices DPR, PRT, CR, K e A (Roush et al., 1989; Hoffman \& Buhler, 2002). Considerou-se como hipótese nula, para testar as diferenças de DPR e A, que as médias fossem iguais a zero $\left(\mathrm{H}_{\mathrm{o}}=0\right)$; para PRT e CR, que as médias fossem iguais à unidade $\left(\mathrm{H}_{\mathrm{o}}=1\right)$; e, para $\mathrm{K}$, que as médias das diferenças entre $\mathrm{K}_{\mathrm{x}}$ e $\mathrm{K}_{\mathrm{y}}$ fossem iguais a zero $\left[\mathrm{H}_{\mathrm{o}}=\right.$ $\left.\left(\mathrm{K}_{\mathrm{x}}-\mathrm{K}_{\mathrm{y}}\right)=0\right]$. $\mathrm{O}$ critêrio para considerar as curvas de PR e PRT diferentes das retas hipotéticas foi que, no mínimo em duas proporções, ocorressem diferenças significativas pelo teste " $t$ ". Do mesmo modo, considerou-se, para os indices $\mathrm{CR}, \mathrm{K}$ e A, a existência de diferenças em competitividade quando, no mínimo em dois deles, houvesse diferenças significativas pelo teste "t" (Bianchi et al., 2006).

Os resultados obtidos para Afi, Est, AF e MS, expressos em valores médios por planta, foram submetidos à análise de variância $(\mathrm{p} \leq 0,05)$. Em caso de significância, as médias dos tratamentos foram comparadas pelo teste de Dunnett $(\mathrm{p} \leq 0,05)$, considerando-se as monoculturas como testemunhas nessas comparações.

\section{RESULTADOS E DISCUSSÃO}

Os resultados observados na combinação do cultivar IRGA 417 com o simulador de arroz-vermelho (EEA 406) demonstraram que os valores de PR esperados foram muito próximos aos observados, em todas as variáveis estudadas (Figuras 1, 2, 3 e 4). Em todas as situações não houve diferenças significativas para PR,

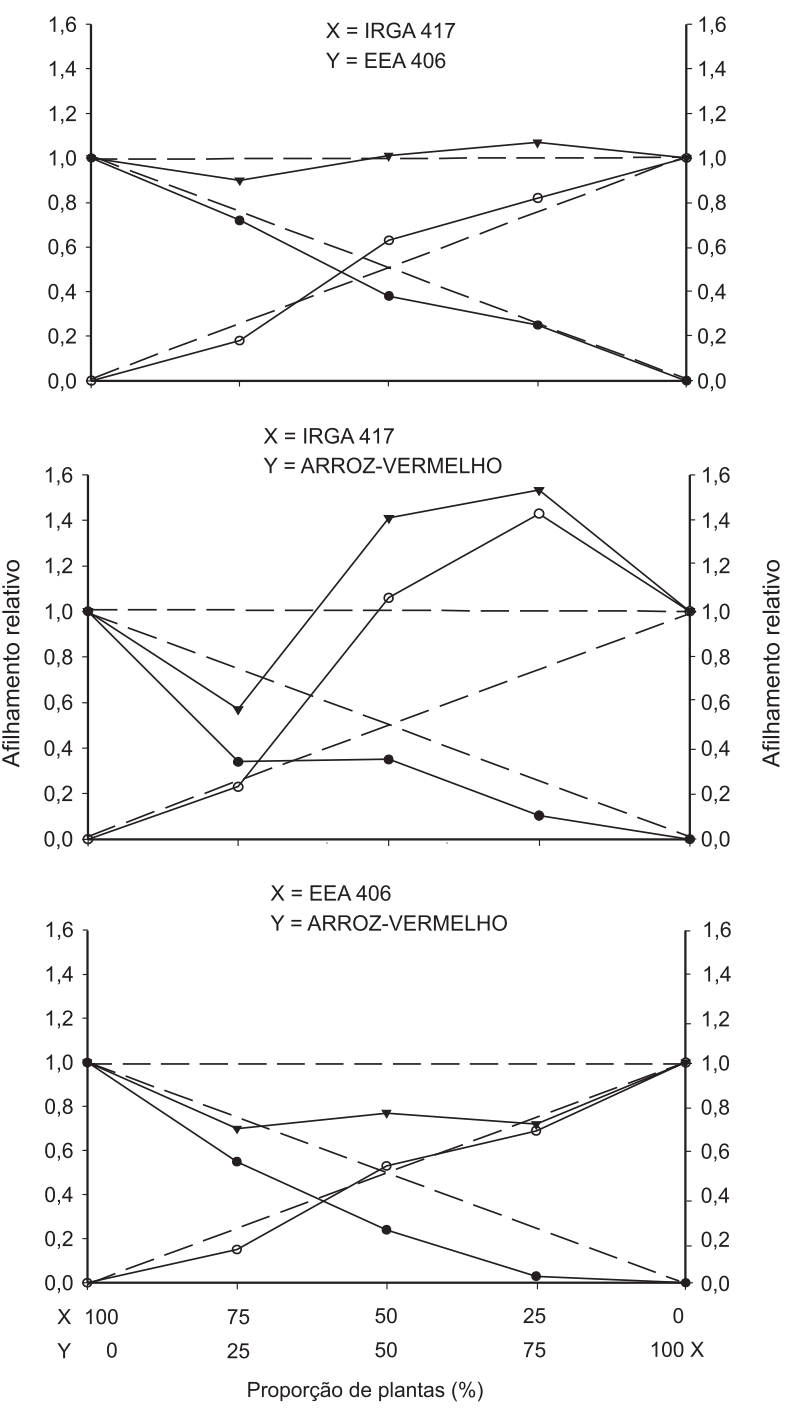

Figura 1 - Produtividade relativa (PR) e total (PRT) para afilhamento de plantas de arroz e arroz-vermelho, UFRGS, Porto Alegre/RS, 2001/02. (•) PR do cultivar de arroz (X), (o) PR do competidor (Y) e ( $\mathbf{\nabla})$ PRT. Linhas tracejadas referem-se às produtividades relativas hipotéticas, quando não ocorre interferência de uma espécie sobre a outra.

com exceção de Afi da cultura e de AF do competidor (Figuras 1 e 3; Tabela 1). Com relação à PRT, em geral, não houve diferença estatística entre os valores esperados e estimados para todas as variáveis estudadas, tendo estas apresentado valores médios próximos à unidade em todas as combinações (Figuras 1, 2, 3 e 4; Tabela 1).

A análise gráfica das combinações de plantas do cultivar IRGA 417 e do biótipo de arroz- 

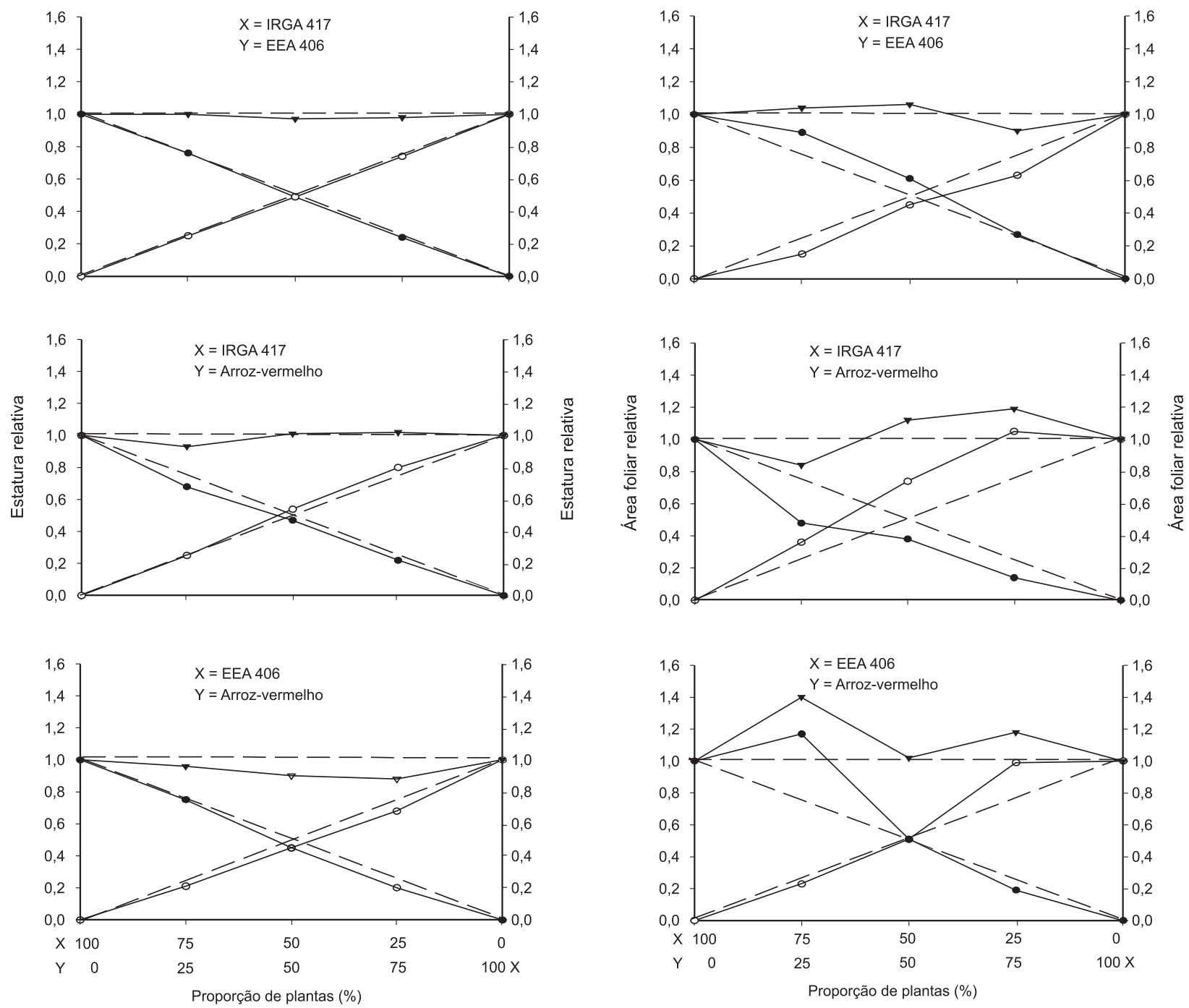

Figura 2 - Produtividade relativa (PR) e total (PRT) para estatura de plantas de arroz e arroz-vermelho, UFRGS, Porto Alegre/RS, 2001/02. (•) PR do cultivar de arroz (X), (o) PR do competidor (Y) e ( $)$ PRT. Linhas tracejadas referem-se às produtividades relativas hipotéticas, quando não ocorre interferência de uma espécie sobre a outra.

vermelho, para todas as variáveis, mostrou que os desvios observados das retas da PR, em relação às retas esperadas, são representados por linhas côncavas para a cultura e por linhas convexas para o competidor (Figuras 1, 2, 3 e 4), demonstrando que a cultura é menos competitiva que o arroz-daninho. Considerando que, para haver significância, pelo menos duas proporções de plantas devem apresentar diferenças significativas (Bianchi et al., 2006), não

se observaram diferenças entre as retas estimadas e as esperadas para o cultivar IRGA 417, para a variável Est (Figuras 1, 3 e 4; Tabela 1).

Com relação à PRT da combinação, para todas as variáveis não se constataram diferenças, mas, de modo geral, seus valores foram equivalentes à unidade (Tabela 1). Isso demonstra que os biótipos interagem pelos 

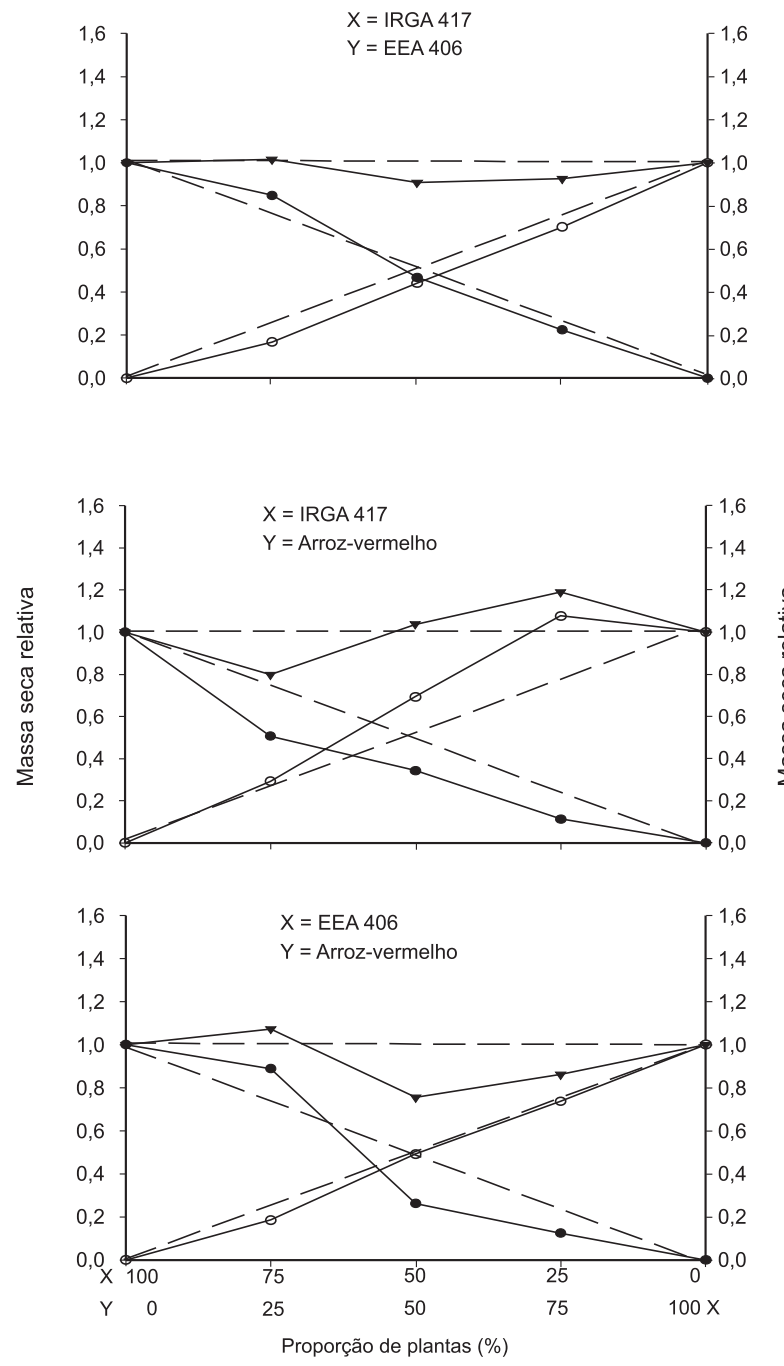

Figura 4 - Produtividade relativa (PR) e total (PRT) para massa seca de plantas de arroz e arroz-vermelho, UFRGS, Porto Alegre/RS, 2001/02. (•) PR do cultivar de arroz (X), (o) PR do competidor (Y) e ( $)$ PRT. Linhas tracejadas referem-se às produtividades relativas hipotéticas, quando não ocorre interferência de uma espécie sobre a outra.

mesmos recursos disponiveis no ambiente, mas um utiliza os recursos do meio de forma mais eficiente que outro. Esse comportamento mostra que um biótipo é mais competitivo que o outro e contribui mais que o esperado para a produtividade total da associação (Radosevich, 1987).

A interpretação dos resultados gráficos da combinação do cultivar EEA 406 com o biótipo de arroz-vermelho revela que, em geral, tanto a cultura quanto o competidor apresentaram valores de PR inferiores aos das retas estimadas, apesar de não ocorrer significância para a variável AF do cultivar (Figuras 1, 2 e 4; Tabela 1). Já para PRT, observou-se, em todas as simulações, que ela foi inferior à unidade, mas ocorreram diferenças entre os valores dos desvios esperados e os observados somente para MS (Figura 4; Tabela 1). Assim, constatou-se que houve competição pelos mesmos recursos do ambiente e na mesma proporção, ocorrendo antagonismo entre as espécies, sem alteração na produtividade final (Cousens, 1991).

De modo geral, o crescimento relativo do cultivar IRGA 417 sempre foi menor quando a participação do competidor era de $75 \%$ (arrozvermelho ou EEA 406) para $25 \%$ da cultura (Figuras 1, 2, 3 e 4; Tabela 1). A provável causa de os competidores apresentarem maior crescimento naquela proporção pode estar relacionada à estatura de plantas, tornando-os mais eficientes na captura da luz solar e impondo sombreamento à cultura (Garrity et al., 1992). Deve-se ressaltar que, em experimentos substitutivos, existe pouca evidência de haver mudanças qualitativas devido ao aumento da população, ou seja, a dominância de uma espécie sobre a outra raramente muda com a alteração da população (Cousens \& O'Neill, 1993).

Em relação aos competidores, observou-se, em todas as combinações (exceto para o cultivar IRGA 417 na presença do simulador de arroz-vermelho (EEA 406) para a variável AF), que não houve diferenças entre arroz-vermelho ou EEA 406 em competição com o arroz cultivado (Tabela 1). Isso demonstra que o arroz-vermetho e seu cultivar simulador apresentam características morfofisiológicas semelhantes e que as plantas possuem habilidades competitivas equivalentes.

Para as variáveis morfológicas estudadas, reduções significativas foram observadas quando os cultivares IRGA 417 e EEA 406 competiram com o arroz-vermelho, sobretudo quando houve predominio de plantas de arroz-vermelho na associação (proporção 75:25) (Tabela 2). Para o cultivar IRGA 417, mesmo nas menores proporções do arroz-vermelho (25:75), observouse interferência sobre o cultivar, reduzindo as variáveis Afi, AF e MS. Com relação aos genótipos competidores, não foram verificadas 
Competitividade relativa entre cultivares de arroz irrigado e...

Tabela 1 - Diferenças de produtividade relativa (DPR) e de produtividade relativa total (PRT), nas proporções 75:25, 50:50 e 25:75 de cultivares de arroz (IRGA 417 ou EEA 406) e de competidores (EEA 406 ou arroz-vermelho), aos 32 dias após a emergência do arroz. UFRGS, Porto Alegre-RS, 2001/02

\begin{tabular}{|c|c|c|c|}
\hline \multirow{2}{*}{ Variável } & \multicolumn{3}{|c|}{ Proporção de planta associada (arroz:competidor) } \\
\hline & $75: 25$ & $50: 50$ & $25: 75$ \\
\hline \multicolumn{4}{|c|}{ Afilhamento } \\
\hline $\begin{array}{l}\text { DPR IRGA } 417 \\
\text { DPR EEA } 406^{2} \\
\text { PRT }\end{array}$ & $\begin{array}{c}-0,03( \pm 0,06)^{\mathrm{ns}} \\
0,07( \pm 0,05)^{\mathrm{ns}} \\
0,90( \pm 0,08)^{\mathrm{ns}}\end{array}$ & $\begin{array}{c}-0,12( \pm 0,03)^{*} \\
0,13( \pm 0,03)^{*} \\
1,01( \pm 0,05)^{\mathrm{ns}}\end{array}$ & $\begin{array}{r}-0,05( \pm 0,02)^{*} \\
-0,07( \pm 0,03)^{\mathrm{ns}} \\
1,06( \pm 0,07)^{\mathrm{ns}}\end{array}$ \\
\hline $\begin{array}{l}\text { DPR IRGA } 417 \\
\text { DPR Arroz-vermelho }{ }^{2} \\
\text { PRT }\end{array}$ & $\begin{array}{c}-0,41( \pm 0,06)^{*} \\
-0,02( \pm 0,02)^{\mathrm{ns}} \\
0,57( \pm 0,07)^{*}\end{array}$ & $\begin{array}{c}-0,15( \pm 0,10)^{\mathrm{ns}} \\
0,56( \pm 0,22)^{\mathrm{ns}} \\
1,41( \pm 0,31)^{\mathrm{ns}}\end{array}$ & $\begin{array}{c}-0,15( \pm 0,02)^{*} \\
0,68( \pm 0,18)^{*} \\
1,53( \pm 0,20)^{\mathrm{ns}}\end{array}$ \\
\hline $\begin{array}{l}\text { DPR EEA } 406 \\
\text { DPR Arroz-vermelho } \\
\text { PRT }\end{array}$ & $\begin{array}{c}-0,20( \pm 0,06)^{\mathrm{ns}} \\
-0,05( \pm 0,11)^{\mathrm{ns}} \\
0,71( \pm 0,05)^{*}\end{array}$ & $\begin{array}{c}-0,26( \pm 0,07)^{*} \\
0,03( \pm 0,08)^{\mathrm{ns}} \\
0,77( \pm 0,12)^{\mathrm{ns}}\end{array}$ & $\begin{array}{c}-0,21( \pm 0,02)^{*} \\
-0,10( \pm 0,04)^{n s} \\
0,73( \pm 0,12)^{n s}\end{array}$ \\
\hline \multicolumn{4}{|c|}{ Estatura } \\
\hline $\begin{array}{l}\text { DPR IRGA } 417 \\
\text { DPR EEA } 406^{2} \\
\text { PRT }\end{array}$ & $\begin{array}{c}0,01( \pm 0,02)^{\mathrm{ns}} \\
-0,01( \pm 0,01)^{*} \\
1,00( \pm 0,02)^{\mathrm{ns}}\end{array}$ & $\begin{array}{l}-0,01( \pm 0,02)^{\mathrm{ns}} \\
-0,01( \pm 0,02)^{\mathrm{ns}} \\
0,97( \pm 0,03)^{\mathrm{ns}}\end{array}$ & $\begin{array}{c}-0,01( \pm 0,02)^{*} \\
-0,01( \pm 0,01)^{\mathrm{ns}} \\
0,98( \pm 0,02)^{*}\end{array}$ \\
\hline $\begin{array}{l}\text { DPR IRGA } 417 \\
\text { DPR Arroz-vermelho } \\
\text { PRT }\end{array}$ & $\begin{array}{l}-0,07( \pm 0,02)^{*} \\
0,05( \pm 0,03)^{\mathrm{ns}} \\
0,93( \pm 0,02)^{\mathrm{ns}}\end{array}$ & $\begin{array}{r}-0,03( \pm 0,01)^{\mathrm{ns}} \\
0,04( \pm 0,01)^{\mathrm{ns}} \\
1,01( \pm 0,01)^{\mathrm{ns}}\end{array}$ & $\begin{array}{c}-0,03( \pm 0,01)^{\mathrm{ns}} \\
0,002( \pm 0,01)^{\mathrm{ns}} \\
1,02( \pm 0,04)^{\mathrm{ns}}\end{array}$ \\
\hline $\begin{array}{l}\text { DPR EEA } 406 \\
\text { DPR Arroz-vermelho } \\
\text { PRT }\end{array}$ & $\begin{array}{c}0,003( \pm 0,02)^{\mathrm{ns}} \\
-0,07( \pm 0,03)^{\mathrm{ns}} \\
0,96( \pm 0,03)^{\mathrm{ns}}\end{array}$ & $\begin{array}{c}-0,05( \pm 0,01)^{*} \\
-0,05( \pm 0,02)^{\mathrm{ns}} \\
0,90( \pm 0,02)^{*}\end{array}$ & $\begin{array}{l}-0,05( \pm 0,01)^{*} \\
-0,04( \pm 0,01)^{\mathrm{ns}} \\
0,88( \pm 0,04)^{\mathrm{ns}}\end{array}$ \\
\hline \multicolumn{4}{|c|}{ Área foliar } \\
\hline $\begin{array}{l}\text { DPR IRGA } 417 \\
\text { DPR EEA } 406 \\
\text { PRT }\end{array}$ & $\begin{array}{c}0,14( \pm 0,05)^{\mathrm{ns}} \\
-0,12( \pm 0,02)^{*} \\
1,04( \pm 0,06)^{\mathrm{ns}}\end{array}$ & $\begin{array}{c}0,11( \pm 0,03)^{*} \\
-0,05( \pm 0,04)^{\mathrm{ns}} \\
1,05( \pm 0,06)^{\mathrm{ns}}\end{array}$ & $\begin{array}{r}0,02( \pm 0,02)^{\mathrm{ns}} \\
-0,10( \pm 0,03)^{*} \\
0,90( \pm 0,03)^{\mathrm{ns}}\end{array}$ \\
\hline $\begin{array}{l}\text { DPR IRGA } 417 \\
\text { DPR Arroz-vermelho } \\
\text { PRT }\end{array}$ & $\begin{array}{c}-0,27( \pm 0,02)^{*} \\
0,30( \pm 0,12)^{\mathrm{ns}} \\
0,84( \pm 0,04)^{*}\end{array}$ & $\begin{array}{c}-0,12( \pm 0,06)^{\mathrm{ns}} \\
0,24( \pm 0,09)^{\mathrm{ns}} \\
1,12( \pm 0,13)^{\mathrm{ns}}\end{array}$ & $\begin{array}{l}-0,11( \pm 0,01)^{*} \\
0,11( \pm 0,04)^{\mathrm{ns}} \\
1,19( \pm 0,13)^{\mathrm{ns}}\end{array}$ \\
\hline $\begin{array}{l}\text { DPR EEA } 406 \\
\text { DPR Arroz-vermelho } \\
\text { PRT }\end{array}$ & $\begin{array}{l}0,42( \pm 0,16)^{\mathrm{ns}} \\
0,24( \pm 0,11)^{\mathrm{ns}} \\
1,41( \pm 0,19)^{\mathrm{ns}}\end{array}$ & $\begin{array}{l}0,01( \pm 0,03)^{\mathrm{ns}} \\
0,01( \pm 0,08)^{\mathrm{ns}} \\
1,02( \pm 0,08)^{\mathrm{ns}}\end{array}$ & $\begin{array}{r}-0,06( \pm 0,05)^{\mathrm{ns}} \\
-0,02( \pm 0,04)^{\mathrm{ns}} \\
1,18( \pm 0,10)^{\mathrm{ns}}\end{array}$ \\
\hline \multicolumn{4}{|c|}{ Massa seca aérea } \\
\hline $\begin{array}{l}\text { DPR IRGA } 417 \\
\text { DPR EEA } 406 \\
\text { PRT }\end{array}$ & $\begin{array}{c}0,10( \pm 0,02)^{*} \\
-0,05( \pm 0,03)^{\mathrm{ns}} \\
1,01( \pm 0,05)^{\mathrm{ns}}\end{array}$ & $\begin{array}{c}-0,03( \pm 0,02)^{\mathrm{ns}} \\
-0,06( \pm 0,02)^{*} \\
0,91( \pm 0,01)^{*}\end{array}$ & $\begin{array}{l}-0,03( \pm 0,02)^{\mathrm{ns}} \\
-0,08( \pm 0,03)^{\mathrm{ns}} \\
0,93( \pm 0,05)^{\mathrm{ns}}\end{array}$ \\
\hline $\begin{array}{l}\text { DPR IRGA } 417 \\
\text { DPR Arroz-vermelho } \\
\text { PRT }\end{array}$ & $\begin{array}{r}-0,24( \pm 0,02)^{*} \\
0,33( \pm 0,03)^{*} \\
0,80( \pm 0,04)^{*}\end{array}$ & $\begin{array}{c}-0,16( \pm 0,04)^{*} \\
0,19( \pm 0,08)^{\mathrm{ns}} \\
1,04( \pm 0,08)^{\mathrm{ns}}\end{array}$ & $\begin{array}{c}-0,14( \pm 0,01)^{*} \\
0,04( \pm 0,03)^{\mathrm{ns}} \\
1,19( \pm 0,03)^{*}\end{array}$ \\
\hline $\begin{array}{l}\text { DPR EEA } 406 \\
\text { DPR Arroz-vermelho } \\
\text { PRT }\end{array}$ & $\begin{array}{r}0,14( \pm 0,14)^{\mathrm{ns}} \\
-0,01( \pm 0,04)^{\mathrm{ns}} \\
1,07( \pm 0,13)^{\mathrm{ns}}\end{array}$ & $\begin{array}{c}-0,24( \pm 0,03)^{*} \\
-0,01( \pm 0,03)^{\mathrm{ns}} \\
0,75( \pm 0,04)^{*}\end{array}$ & $\begin{array}{c}-0,13( \pm 0,01)^{*} \\
-0,06( \pm 0,02)^{\mathrm{ns}} \\
0,86( \pm 0,04)^{*}\end{array}$ \\
\hline
\end{tabular}

* Diferença significativa pelo teste $\mathrm{t}(\mathrm{p} \leq 0,05)$. Valores entre parênteses representam os erros-padrão das médias. 
Tabela 2 - Resposta de cultivares de arroz (IRGA 417 ou EEA 406) à interferência de competidores (EEA 406 ou arroz-vermelho), em experimentos conduzidos em séries substitutivas, aos 32 dias após a emergência. UFRGS, Porto Alegre-RS, 2001/02

\begin{tabular}{|c|c|c|c|c|}
\hline $\begin{array}{l}\text { Proporção de planta } \\
\text { (Arroz:competidor) }\end{array}$ & $\begin{array}{c}\text { Afilho } \\
\left(\mathrm{n}^{\mathrm{o}} \text { planta }^{-1}\right)\end{array}$ & $\begin{array}{l}\text { Estatura } \\
(\mathrm{cm})\end{array}$ & $\begin{array}{c}\text { Área foliar } \\
\left(\mathrm{cm}^{2} \text { planta }^{-1}\right)\end{array}$ & $\begin{array}{l}\text { Massa seca } \\
\left(\mathrm{g}_{\text {planta }}{ }^{-1}\right)\end{array}$ \\
\hline \multicolumn{5}{|c|}{ Cultivar IRGA 417} \\
\hline $100: 0(\mathrm{~T})$ & 2,7 & 58,2 & 79,7 & 0,9 \\
\hline $75: 25$ & 2,6 & 58,8 & 95,0 & 1,0 \\
\hline $50: 50$ & 2,1 & 56,8 & 96,7 & 0,8 \\
\hline $25: 75$ & 2,6 & 55,8 & 84,6 & 0,8 \\
\hline $\mathrm{CV}(\%)$ & 16,2 & 4,4 & 12,7 & 11,4 \\
\hline \multicolumn{5}{|c|}{ Competidor EEA 406} \\
\hline $0: 100(\mathrm{~T})$ & 1,7 & 63,3 & 115,2 & 1,0 \\
\hline $25: 75$ & 1,8 & 62,3 & 97,1 & 0,9 \\
\hline $50: 50$ & 2,1 & 61,4 & 102,7 & 0,8 \\
\hline $75: 25$ & 1,2 & 62,4 & $67,7^{*}$ & $0,6^{*}$ \\
\hline $\mathrm{CV}(\%)$ & 17,9 & 5,9 & 18,9 & 16,6 \\
\hline \multicolumn{5}{|c|}{ Cultivar IRGA 417} \\
\hline $100: 0(\mathrm{~T})$ & 2,8 & 65,6 & 91,4 & 1,2 \\
\hline $75: 25$ & $1,3^{*}$ & 59,5 & $58,0^{*}$ & $0,8^{*}$ \\
\hline $50: 50$ & 2,0 & 62,3 & 69,1 & $0,8^{*}$ \\
\hline $25: 75$ & $1,2 *$ & $56,4^{*}$ & $50,5^{*}$ & $0,5^{*}$ \\
\hline $\mathrm{CV}(\%)$ & 36,7 & 7,2 & 19,5 & 13,5 \\
\hline \multicolumn{5}{|c|}{ Competidor arroz-vermelho } \\
\hline $0: 100(\mathrm{~T})$ & 1,0 & 73,0 & 81,0 & 0,9 \\
\hline $25: 75$ & 1,9 & 78,0 & 113,4 & 1,2 \\
\hline $50: 50$ & $2,1 *$ & 78,6 & 119,3 & 1,2 \\
\hline $75: 25$ & 0,9 & 73,7 & 117,0 & 1,0 \\
\hline $\mathrm{CV}(\%)$ & 35,8 & 6,0 & 22,2 & 17,1 \\
\hline \multicolumn{5}{|c|}{ Cultivar EEA 406} \\
\hline $100: 0(\mathrm{~T})$ & 1,8 & 73,5 & 49,8 & 0,8 \\
\hline $75: 25$ & 1,3 & 73,8 & 77,8 & 1,0 \\
\hline $50: 50$ & $0,8^{*}$ & 66,0 & 50,3 & $0,4 *$ \\
\hline $25: 75$ & $0,3 *$ & $59,2 *$ & 38,7 & $0,4^{*}$ \\
\hline $\mathrm{CV}(\%)$ & 37,7 & 6,1 & 27,5 & 25,0 \\
\hline \multicolumn{5}{|c|}{ Competidor arroz-vermelho } \\
\hline $0: 100(\mathrm{~T})$ & 2,3 & 86,6 & 102,7 & 1,4 \\
\hline $25: 75$ & 2,1 & 78,0 & 135,5 & 1,3 \\
\hline $50: 50$ & 2,4 & 78,5 & 105,0 & 1,3 \\
\hline $75: 25$ & 1,4 & $73,0 *$ & 96,0 & 1,0 \\
\hline $\mathrm{CV}(\%)$ & 30,5 & 8,8 & 34,1 & 15,4 \\
\hline
\end{tabular}

* Média difere da testemunha $(\mathrm{T})$ pelo teste de Dunnett $(\mathrm{p} \leq 0,05) . \mathrm{CV}=$ coeficiente de variação. 
variáveis morfológicas alteradas, independentemente da proporção populacional em que participaram nas associações. Contudo, excetuam-se: Afi de arroz-vermelho associado com IRGA 417 na proporção 50:50; Est para o mesmo competidor em associação com o cultivar EEA 406 na proporção 75:25; e AF e MS do genótipo competidor EEA 406 associado com IRGA 417 na proporção 75:25.

De modo geral, observou-se que o arrozvermelho, ao competir com os cultivares de arroz EEA 406 e IRGA 417, apresentou maior crescimento que este em todas as variáveis avaliadas, conforme indicado pelo índice CR, exceto a variável AF quando o cultivar IRGA 417 competiu com o simulador EEA 406 (Tabela 3). No entanto, ocorreu dominância dos competidores sobre os cultivares de arroz, principalmente quando o competidor foi o biótipo de arroz-vermelho, em ambos os cultivares (IRGA 417 ou EEA 406), conforme indicado pelo indice K. Constatou-se ainda que o arrozvermelho também foi mais competitivo que o genótipo EEA 406 - resposta apontada pelo indice A. Ressalta-se que, em algumas situações, não houve diferenças significativas para cultivares ou competidores, demonstrando que ambos se equivaleram em termos de competição pelos recursos do meio.

Considera-se que um genótipo $\mathrm{X}$ é mais competitivo que um $\mathrm{Y}$ quando $\mathrm{CR}>1, \mathrm{~K}_{\mathrm{x}}>\mathrm{K}_{\mathrm{y}}$ e A > 0 (Hoffman \& Buhler, 2002). Adotou-se como critério para comprovar superioridade competitiva a ocorrência de diferenças significativas em pelo menos dois índices (Bianchi et al., 2006). Assim, o biótipo de arroz-vermelho foi mais competitivo que os cultivares de arroz IRGA 417 e EEA 406, para todas as variáveis avaliadas, com exceção da AF de EEA 406 quando competiu com arroz-vermelho (Tabela 3). Isso demonstra que o arroz-vermelho é realmente muito competitivo, pois superou seu simulador (EEA 406), apesar de este apresentar características morfofisiológicas próximas às do arrozvermelho, sendo elevado o dano que aquele ocasiona (Pabón, 1990). Utilizando conjuntamente os três índices para definir competitividade, Hoffman \& Buhler (2002) verificaram que o sorgo cultivado foi mais competitivo que Sorghum halepense; e Bianchi et al. (2006) constataram que nabo forrageiro foi mais competitivo que genótipos de soja.
Ao explorarem basicamente o mesmo nicho ecológico, os genótipos de arroz competem pelos mesmos recursos no tempo e/ou no espaço. Assim, verificou-se que houve diferenças em termos de competitividade dos genótipos estudados. De igual forma, Pantone \& Baker (1991) observaram comportamento semelhante ao estudaram a competição entre arroz e arroz-vermelho, assim como Hoffman \& Buhler (2002), ao estudarem associações de sorgo e sorgo-de-alepo ou sorgo e biótipo silvestre originado do sorgo cultivado.

Interpretando conjuntamente as análises gráficas das variáveis relativas, bem como suas significâncias em relação aos valores equivalentes às variáveis morfológicas e aos indices de competitividade, em geral, foram constatados efeitos de competição do arrozvermelho sobre o cultivar de arroz IRGA 417. Ao explorar o mesmo nicho ecológico do arrozdaninho, o cultivar IRGA 417 foi mais prejudicado pela competição. Provavelmente isso se deve à maior capacidade competitiva do arrozvermelho (Pantone \& Baker, 1991), especialmente por luz, pois apresenta maior estatura do que o arroz cultivado (Agostinetto et al., 2001).

De modo geral, as diferenças morfofisiológicas existentes entre plantas dos dois cultivares de arroz (IRGA 417 e EEA 406) não foram suficientes para demonstrar, durante a execução da pesquisa, competição diferencial por recursos do ambiente e, conseqüentemente, caracterizar variações expressivas em competitividade entre os referidos genótipos. Quando o arroz-vermelho competiu com o genótipo IRGA 417, não foi possível identificar o recurso pelo qual ambos competiram. Presume-se que a interferência tenha ocorrido principalmente por luz, mas não se pode descartar o fato de o arroz-vermelho ter conseguido capturar com maior eficiência nutrientes e água do solo. De acordo com Agostinetto et al. (2004), o arrozvermelho apresenta maior crescimento, em relação a diferentes cultivares de arroz, quando a adubação nitrogenada é totalmente aplicada na semeadura da cultura.

Os resultados mostraram que o arroz-vermelho modificou negativamente o número de afilhos, estatura, área foliar e massa seca da parte aérea de plantas dos cultivares IRGA 417 e EEA 406, demonstrando superioridade 
Tabela 3 - Índices de competitividade entre cultivares de arroz (IRGA 417 ou EEA 406) e competidores (EEA 406 ou arrozvermelho), expressos por competitividade relativa (CR), coeficientes de agrupamento relativo (K) e de agressividade (A), obtidos em experimentos conduzidos em séries substitutivas. UFRGS, Porto Alegre-RS, 2001/02

\begin{tabular}{|c|c|c|c|c|}
\hline Variável & $\mathrm{CR}$ & $\mathrm{K}_{\mathrm{x}}(\operatorname{arroz})$ & $\mathrm{K}_{\mathrm{y}}$ (competidor) & A \\
\hline \multicolumn{5}{|c|}{ Afilhamento } \\
\hline IRGA 417 x EEA 406 & $0,62( \pm 0,06)^{*}$ & $0,64( \pm 0,10)$ & $1,74( \pm 0,23)^{*}$ & $-0,24( \pm 0,05)^{*}$ \\
\hline IRGA $417 \mathrm{x}$ arroz-vermelho & $0,32( \pm 0,06)^{*}$ & $0,65( \pm 0,24)$ & $1,69( \pm 2,01)$ & $-0,71( \pm 0,14)^{*}$ \\
\hline EEA $406 \mathrm{x}$ arroz-vermelho & $0,45( \pm 0,15)^{*}$ & $0,35( \pm 0,14)$ & $1,08( \pm 0,54)$ & $-0,30( \pm 0,09)^{*}$ \\
\hline \multicolumn{5}{|c|}{ Estatura } \\
\hline IRGA 417 x EEA 406 & $1,01( \pm 0,01)$ & $0,96( \pm 0,07)$ & $0,95( \pm 0,06)$ & $0,003( \pm 0,01)$ \\
\hline IRGA $417 \mathrm{x}$ arroz-vermelho & $0,88( \pm 0,03)^{*}$ & $0,91( \pm 0,04)$ & $1,17( \pm 0,06)^{*}$ & $-0,06( \pm 0,02)^{*}$ \\
\hline EEA $406 \mathrm{x}$ arroz-vermelho & $0,99( \pm 0,02)$ & $0,82( \pm 0,03)$ & $0,83( \pm 0,05)$ & $-0,004( \pm 0,01)$ \\
\hline \multicolumn{5}{|c|}{ Área foliar } \\
\hline IRGA 417 x EEA 406 & $1,39( \pm 0,14)$ & $1,59( \pm 0,19)$ & $0,84( \pm 0,16)^{*}$ & $0,16( \pm 0,05)^{*}$ \\
\hline IRGA $417 x$ arroz-vermelho & $0,51( \pm 0,03)^{*}$ & $0,47( \pm 0,02)$ & $1,89( \pm 0,17)^{*}$ & $-0,36( \pm 0,04)^{*}$ \\
\hline EEA $406 \mathrm{x}$ arroz-vermelho & $1,06( \pm 0,17)$ & $1,04( \pm 0,11)$ & $0,79( \pm 0,08)$ & $-0,01( \pm 0,09)$ \\
\hline \multicolumn{5}{|c|}{ Massa seca da parte aérea } \\
\hline IRGA 417 x EEA 406 & $1,07( \pm 0,10)$ & $0,89( \pm 0,10)$ & $0,79( \pm 0,05)$ & $0,03( \pm 0,04)$ \\
\hline IRGA $417 \mathrm{x}$ arroz-vermelho & $0,52( \pm 0,08)^{*}$ & $0,54( \pm 0,11)$ & $1,60( \pm 0,12)^{*}$ & $-0,35( \pm 0,10)^{*}$ \\
\hline EEA $406 \mathrm{x}$ arroz-vermelho & $0,54( \pm 0,06)^{*}$ & $0,36( \pm 0,05)$ & $0,99( \pm 0,11)^{*}$ & $-0,23( \pm 0,04)^{*}$ \\
\hline
\end{tabular}

* Diferença significativa pelo teste $\mathrm{t}(\mathrm{p} \leq 0,05)$. Valores entre parênteses representam os erros-padrão das médias. $\mathrm{K}_{\mathrm{x}}$ e $\mathrm{K}_{\mathrm{y}}$ são os coeficientes de agrupamento relativo.

competitiva. O cultivar de arroz IRGA 417 não modifica suas características morfofisiológicas quando em competição com o cultivar EEA 406, independentemente da proporção de plantas. O cultivar EEA 406, por sua vez, também não alterou as variáveis morfológicas quando associado ao cultivar IRGA 417.

\section{LITERATURA CITADA}

AGOSTINETTO, D. et al. Arroz vermelho: ecofisiologia e estratégias de controle. Ci. Rural, v. 31, n. 2, p. 341-349, 2001.

AGOSTINETTO, D. et al. Influência de cultivares de arroz e épocas da adubação nitrogenada nas relações de interferência da cultura com cultivar simulador de infestação de arrozvermelho. Planta Daninha, v. 22, n. 2, p.185-193, 2004

BIANCHI, M. A; FLECK, N. G.; LAMEGO, F. P. Proporção entre plantas de soja e plantas competidoras e as relações de interferência mútua. Ci. Rural, v. 36, n. 5, p.1380-1387, 2006

CHRISTOFFOLETI, P. J.; VICTORIA FILHO, R. Efeitos da densidade e proporção de plantas de milho (Zea mays L.) e caruru (Amaranthus retroflexus L.) em competição. Planta Daninha, v. 14, n. 1, p. 42-47, 1996
COMPANHIA NACIONAL DE ABASTECIMENTO CONAB. Arroz - Brasil. Série histórica de: área, produtividade e produção. Disponível em: $<\mathrm{http}$ :// www.conab.gov.br>. Acesso em: 5 de out. de 2006 .

COUSENS, R. Aspects of the design and interpretation of competition (interference) experiments. Weed Technol., v. 5, n. 3, p. 664-673, 1991.

COUSENS, R.; O’NEILL, M. Density dependence of replacement series experiments. Oikos, v. 66, n. 2, p. 347$352,1993$.

EMPRESA BRASILEIRA DE PESQUISA AGROPECUÁRIA - EMBRAPA. Centro Nacional de Pesquisa Agropecuária de Solos (Rio de Janeiro, RJ). Sistema brasileiro de classificação de solos. Brasília: Embrapa Produção de Informação, Rio de Janeiro: Embrapa Solos, 1999. 412p.

FISCHER, A. J.; RAMIREZ, A. Red rice (Oryza sativa): competition studies for management decisions. Inter. J. Pest Manag., v. 39, n. 2, p. 133-138, 1993.

GARRITY, D. P.; MOVILLON, M.; MOODY, K. Differential weed suppression ability in upland rice cultivars. Agron. J., v. 84, n. 4, p. 586-591, 1992. 
HOFFMAN, M. L.; BUHLER, D. D. Utilizing Sorghum as a functional model of crop weed competition. I. Establishing a competitive hierarchy. Weed Sci., v. 50, n. 4, p. 466-472, 2002.

LAMEGO, F. P. et al. Tolerância à interferência de plantas competidoras e habilidade de supressão por genótipos de soja - II. Resposta de variáveis de produtividade. Planta Daninha, v. 22, n. 4, p. 491-498, 2004.

MALUF, A. M. Interferência interespecífica entre Amaranthus hybridus L. e Amaranthus viridis L. Pesq. Agropec. Bras., v. 34, n. 5, p.723-732, 1999.

MENEZES, V. G.; SILVA, P. R. F. Manejo de arroz-vermelho através do tipo e arranjo de plantas em arroz irrigado. Planta Daninha, v. 16, n. 1, p. 45-57, 1998.

OGAWA, M. Red rice. Chem. Organ., v. 30, n. 6, p. $385-$ 388, 1992.

PABÓN, H.A. Principios para el manejo de las malezas en el cultivo del arroz. Comalfi, v. 17, n. 1, p. 28-36, 1990.

PANTONE, D. J.; BAKER, J. B. Reciprocal yield analysis of red rice (Oryza sativa) competition in cultivated rice. Weed Sci., v. 39, n. 1, p. 42-47, 1991.

PASSINI, T. Competitividade e predição de perdas de rendimento da cultura do feijão quando em convivência com Brachiaria plantaginea (Link) Hitchc. 2001. $130 \mathrm{f}$. Tese (Doutorado em Agronomia) - Escola Superior de Agricultura Luiz de Queiroz, 2001.
RADOSEVICH, S. R. Methods to study interactions among crops and weeds. Weed Technol., v. 1, n. 3, p. 190-189, 1987.

ROUSH, M. L. et al. A comparison of methods for measuring effects of density and proportion in plant competition experiments. Weed Sci., v. 37, n. 2, p. 268-275, 1989.

SANCHEZ-OLGUIIN, E.; ARRIETA-ESPINOZA, G.; ESPINOZA ESQUIVEL, A. M. C. Comparação do desenvolvimento vegetativo e reprodutivo do arroz-vermelho e variedades comerciais de arroz (Oriza sativa) da Costa Rica. Planta Daninha, v. 25, n. 1, p. 13-27, 2007.

SOCIEDADE SUL-BRASILEIRA DE ARROZ IRRIGADO - SOSBAI. Arroz irrigado: Recomendações técnicas da pesquisa para o Sul do Brasil. Porto Alegre: IRGA, 2001. $128 \mathrm{p}$.

SOUZA, P. R.; FISCHER, M. M. Arroz vermelho: danos causados à lavoura gaúcha. Lav. Arroz., v. 39, n. 368, p. 1920, 1986.

VAUGHAN, L. K. et al. Is all red rice found in commercial rice really Oryza sativa? Weed Sci., v. 49, n. 4, p. 468-476, 2001.

VILÁ, M.; WILLIAMSON, M.; LONSDALE, M. Competition experiments on alien weeds with crops: lessons for measuring plant invasion impact? Biol. Invasions, v. 6, n. 1, p. 59-69, 2004. 